\title{
Link between the gut and adipose tissues
}

New research suggests that the hepatic enzyme flavin-containing monooxygenase 3 (FMO3) is associated with the beiging of white adipose tissue in mice and humans.

Brown adipose tissue is important for energy expenditure in the form of thermogenesis. Beiging, which is the formation of metabolically active brown adipocytes in white adipose tissue, is thought to be a potential therapeutic strategy to treat obesity. FMO3 is part of the trimethylamine (TMA)-FMO3-TMA N-oxide (TMAO) pathway, a key regulator of lipid metabolism and inflammation. The pathway has an important relationship with the gut, whereby the gut microbiome metabolizes dietary nutrients into TMA, which is further metabolized by FMO3 to produce TMAO.
Previous studies have shown that $\mathrm{FMO} 3$ is involved in the regulation of cholesterol metabolism. In the present study, the authors investigated whether the TMAO pathway is linked to the pathogenesis of obesity. "We found that antisense oligonucleotide-mediated knockdown, as well as genetic deletion, of the TMAO-producing enzyme $\mathrm{FMO} 3$ in mice protects against high-fat diet-induced obesity," explains Mark Brown, corresponding author on the study. The team suggest their findings are, in part, due to the stimulation of beiging, which they believe could have counteracted the adverse effects of increased adiposity.

"Several recent reports have linked the gut microbiome to energy expenditure and cold-induced thermogenic programmes, but the way in which microbes resident in the gut alter adipose tissue biology is poorly understood. Our study provides evidence of a link between the gut and adipose tissue function via the microbe-driven TMA-FMO3-TMAO pathway," says Brown.

The team are now performing preclinical studies examining whether selective TMA lyase inhibitors can prevent the development of obesity or induce weight loss in obese rodents.

Alan Morris

ORIGINAL ARTICLES Schugar, R. C. et al. The TMAO-producing enzyme flavin-containing monooxygenase 3 regulates obesity and the beiging of white adipose tissue.

Cell Rep. 19, 2451-2461 (2017) 